\title{
Error Factors in Oxygenation Measurement Using Continuous Wave and Spatially Resolved Near-infrared Spectroscopy
}

\author{
Masatsugu Niwayama, ${ }^{1}$ Hiroshi Suzuki, ${ }^{1}$ Takayuki Yamashita, ${ }^{1}$ and Yoshifumi Yasuda ${ }^{2}$
}

\begin{abstract}
The main error factors on near-infrared spectroscopy (NIRS) measurements for deep tissue are (1) thickness of overlying tissues, (2) oxygenation of surface tissues, (3) difference between an assumed value and an actual value of scattering coefficient for tissues, and (4) concentrations of hemoglobin derivatives. These factors affect the accuracy on time-resolved NIRS, intensity-modulated NIRS, spatially resolved NIRS, and continuous wave NIRS. In this paper, we present the accurate oxygenation measurement in surface and deep tissues using continuous wave and spatially resolved NIRS. The relationship between spatial distribution of light intensity and absorption coefficient of the muscle was obtained from the Monte Carlo simulation. Fat thickness greatly affected the absolute value of hemoglobin concentration. The influence of skin oxygenation caused several percent error factor of muscle oxygenation. We obtained curves to correct the influences. To reduce the error due to assumed scattering coefficient, a simple method for determining optical properties with laser rangefinder was practical and useful. Moreover, the absolute values of hemoglobin derivative concentrations were successfully measured by spatially resolved NIRS. It was also helpful for accurate oxygenation measurement. The combination of these measurements and corrections would be essential for an accurate NIRS.

(J Jpn Coll Angiol 2012; 52: 211-215)
\end{abstract}

Key words: near-infrared spectroscopy, tissue oxygenation, spatially resolved spectroscopy

\section{Introduction}

Various error factors on near-infrared spectroscopy (NIRS) measurements for deep tissue have been examined theoretically and experimentally, ${ }^{1)}$ and these results suggest that the main factors are (1) thickness of overlying tissues, ${ }^{1}{ }^{\text {) }}$ (2) oxygenation of surface tissues, ${ }^{1,6)}$ (3) difference between an assumed value and an actual value of scattering coefficient for tissues, ${ }^{7)}$ and (4) concentrations of hemoglobin derivatives. ${ }^{8}$ ) These factors affect the accuracy on time-resolved NIRS, intensity-modulated NIRS, spatially resolved NIRS, and continuous wave NIRS. In this paper, we present the methods for correcting the main error factors in order to measure accurate oxygenation in surface and deep tissues using continuous wave NIRS and spatially resolved NIRS.

${ }^{1}$ Department of Electrical and Electronic Engineering, Faculty of Engineering, Shizuoka University, Shizuoka, Japan

${ }^{2}$ Health Science Center, Toyohashi University of Technology,

Aichi, Japan

\section{Influence of Surface Layer Thickness}

The influences of the overlying tissues must be corrected in order to quantify oxygenation using continuous wave NIRS, and a practical correction method to overcome this problem is needed. Figure 1 shows the optical path length in a muscle layer obtained from a Monte Carlo simulation. ${ }^{3,4)}$ The fitting curves at each source-detector separation are shown by normalizing the curves at $0-\mathrm{mm}$ thickness. The relationship between normalized optical path length $S_{\text {muscle }}$ and fat layer thickness $h$ is expressed by the following equation.

$$
S_{\text {muscle }}=\exp \left\{-\left(\frac{h}{A}\right)^{2}\right\}
$$

The values of constant $A$ for the source-detector separations of 20,30 , and $40 \mathrm{~mm}$ are $6.9,8.0$, and 8.9 , respectively. The value of $S_{\text {muscle }}$ can be determined only by $h$ previously

Accepted May 28, 2011 


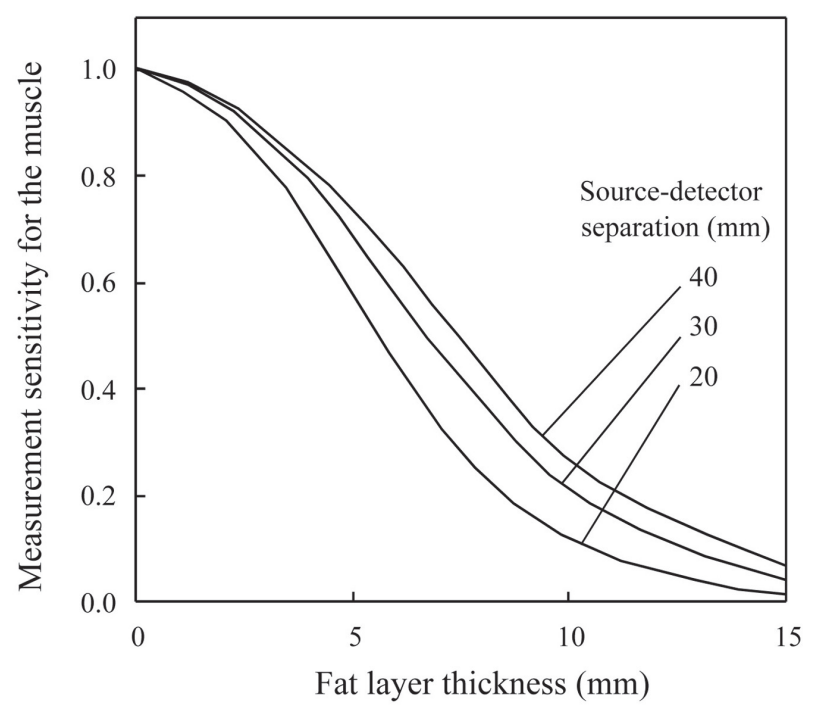

Figure 1 Measurement sensitivity for the muscle on continuous wave single-point NIRS calculated by Monte Carlo simulation.

measured by ultrasonography, and then the changes in hemoglobin concentrations using continuous wave single-point NIRS can be corrected by dividing them by $S_{\text {muscle }}$.

To realize quantitative measurement of the hemoglobin concentrations of the deep tissue with spatially resolved NIRS, we have examined the influence of fat layer thickness on the spatial profile of light intensity. ${ }^{5)}$ Figure 2 shows the increase in spatial slope $S$ due to the increase in the absorption coefficient of the muscle, $\mu_{\mathrm{am}}$, and the increase in the fat layer thickness calculated in the Monte Carlo simulation. The measurements using spatially resolved spectroscopy can be corrected by using the appropriate $S-\mu_{\text {am }}$ curve for fat thickness.

\section{Influence of Skin Oxygenation}

It was difficult to obtain a unique solution on spatially resolved NIRS measurements when both the absorption coefficients of the two adjacent layers are variables. ${ }^{1)}$ In contrast, we have developed a method for simultaneous measurement of $\mu_{\mathrm{as}}$ and $\mu_{\mathrm{am}}$ when there was a low-absorption layer between the muscle and skin layers. ${ }^{6}$ Monte Carlo simulation was used for analyzing light propagation within a three-layered structure composed of skin, fat, and muscle, as shown in Figure 3.

The values $S_{1}$ and $S_{2}$ are the spatial slopes of the light intensity between source-detector distances of 2-4 $\mathrm{mm}$ and 20-30 mm, respectively. We examined the relationship between $\mu_{\text {as }}$ and the spatial slope $S_{1}\left(S_{1}-\mu_{\mathrm{as}}\right.$ curve) and the relationship between $\mu_{\mathrm{am}}$ and the spatial slope $S_{2}\left(S_{2}-\mu_{\text {am }}\right.$ curve $)$. Figure 4 shows the

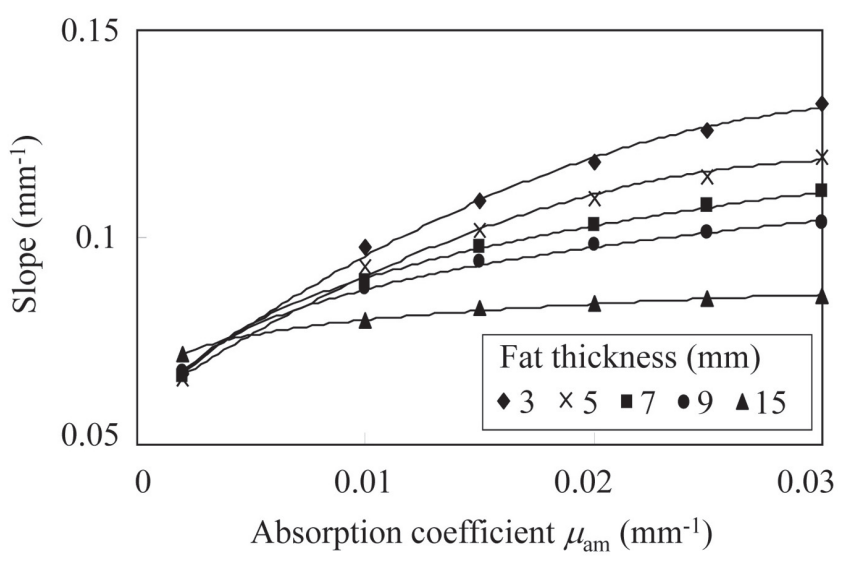

Figure 2 Influence of fat thickness on relationship between slope of light intensity and absorption coefficient of the muscle.

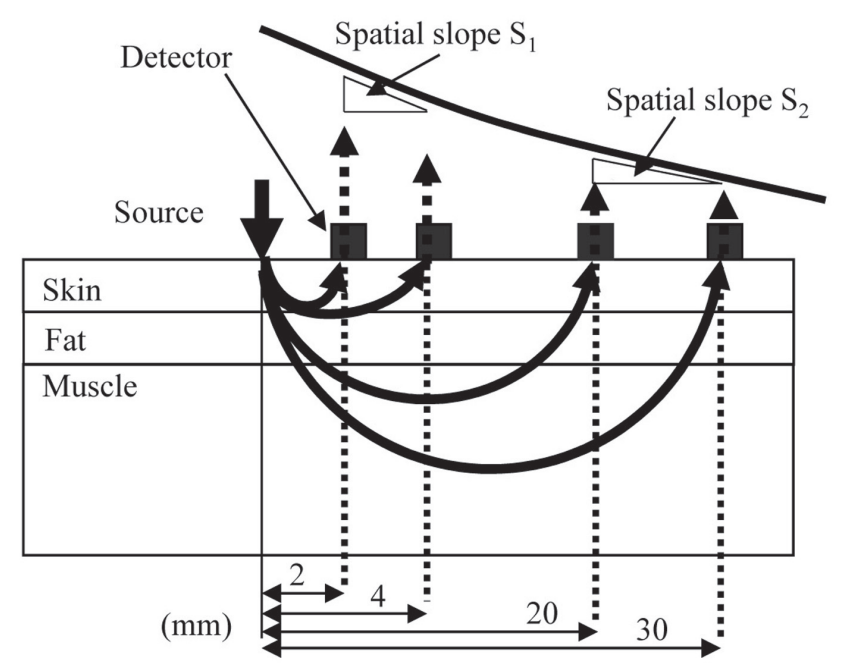

Figure 3 Measurement of two spatial slopes to correct the influence of blood within the skin.

results of the simulation. For example, for $S_{1}=0.61, \mu_{\text {as }}$ can vary from 0.0062 to $0.0476 \mathrm{~mm}^{-1}$ for $\mu_{\mathrm{am}}=0-0.05 \mathrm{~mm}^{-1}$. Similarly, for $S_{2}=0.265, \mu_{\mathrm{am}}$ can vary from 0.0190 to $0.0200 \mathrm{~mm}^{-1}$ for $\mu_{\mathrm{as}}=0-0.05 \mathrm{~mm}^{-1}$. The values for $\mu_{\mathrm{as}}$ and $\mu_{\mathrm{am}}$ were determined by solving the non-linear simultaneous equations obtained by interpolation of the $S_{1}-\mu_{\text {as }}$ and $S_{2}-\mu_{\text {am }}$ curves. Simultaneous determination of absorption coefficients for skin and muscle tissues was examined using the three-layered structure phantom. The base material used was agar, which is optically clear. Black ink (INK-350-B, PILOT) and Intralipid-20\% (Fresenius Kabi AB) were used as absorber and scatter, respectively. The unique solution of the $\mu_{\mathrm{as}}$ and $\mu_{\mathrm{am}}$ pair was obtained for each 


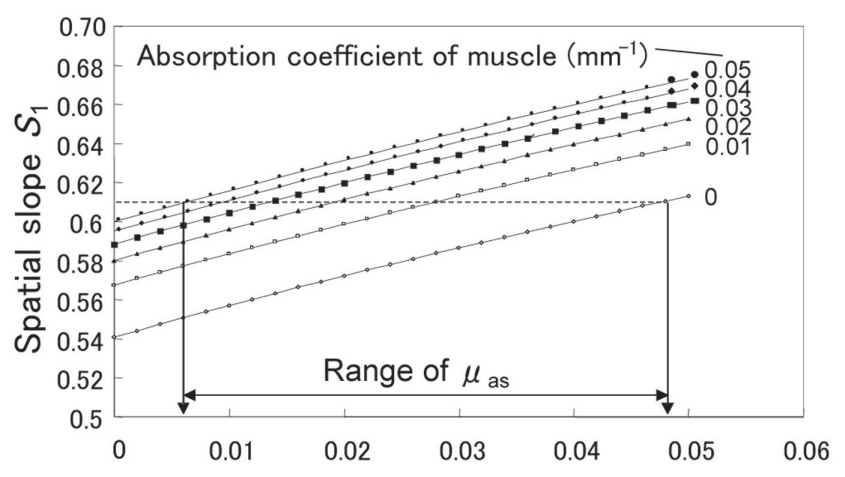

Absorption coefficient of skin $\mu_{\text {as }}\left(\mathrm{mm}^{-1}\right)$

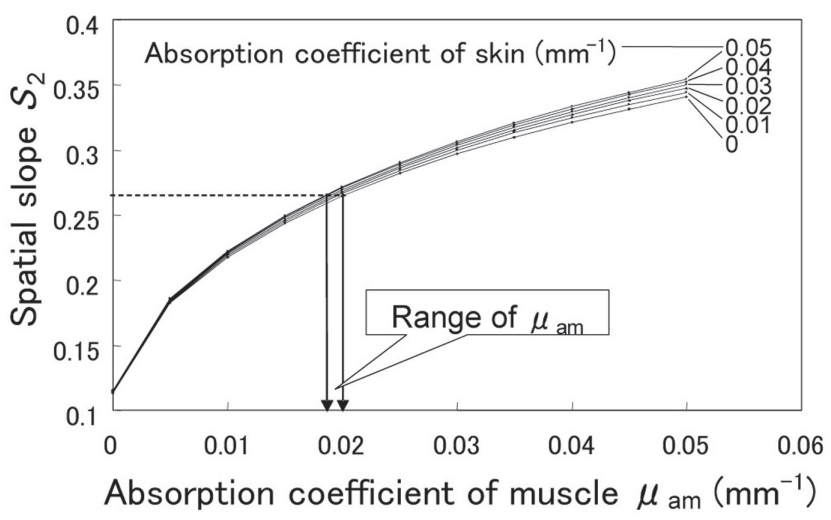

Figure 4 Relationship between (A) absorption coefficient for skin and spatial slope $S_{1}$ and relationship between (B) absorption coefficient for muscle and spatial slope $S_{2}$.

setting value. The actual values of $\mu_{\mathrm{a}}$ of skin and muscle are $0.01-0.02 \mathrm{~mm}^{-1}$. The measurement errors of actual $\mu_{\mathrm{a}}$ range were reduced to within $10 \%$. These methods are useful for reducing influence of the skin on the oxygenation measurement for the muscle.

\section{Influence of Scattering Coefficient of Surface Tissue}

Three methods for obtaining the optical properties are: timeresolved reflectance, intensity-modulated reflectance, and spatially resolved reflectance. The measurement using timeresolved reflectance requires technical expertise, high cost instruments, and a long time for data acquisition. Although the intensity-modulated reflectance method is easy to use, it requires strict noise suppression because of both the amplitude and the phase detection of the high-frequency alternative component. We have combined spatially resolved reflectance with only the phase detection of intensity-modulated reflectance using a laser rangefinder. The measuring system consists of an

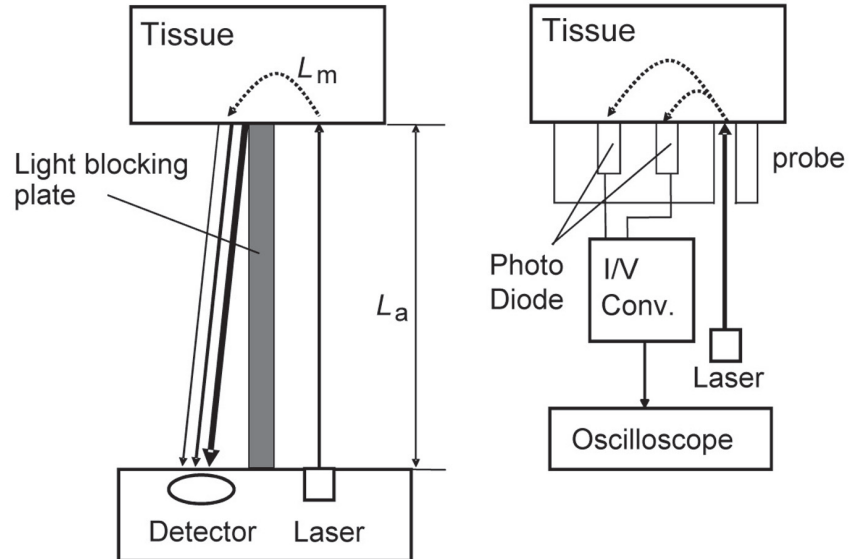

Figure 5 Schematic diagram of the path length measurement (left), and block diagram of the spatially resolved measurement (right).

optical probe for spatially resolved reflectance, a laser rangefinder, and a movable light-blocking plate, as shown in Figure 5. The absorption coefficient $\mu_{\mathrm{a}}$ and the reduced scattering coefficient $\mu_{\mathrm{s}}{ }^{\prime}$ were calculated from the measurements of the optical path length and the spatial profile. Figure 6 shows an example of determination of optical properties at $0.37 \mathrm{~mm}^{-1}$ of slope and $85.5 \mathrm{~mm}$ of path length using the two lookup tables and interpolation. The results of phantom experiments using the proposed system showed that $\mu_{\mathrm{a}}$ and $\mu_{\mathrm{s}}{ }^{\prime}$ can be determined within 5\%-error simultaneously. The developed methods are practical and easy for laboratory use in order to correct the individual difference of scattering coefficient.

\section{Influences of Hemoglobin Derivatives}

Influences of carboxyhemoglobin (COHb) and methemoglobin (MetHb) should be considered for accurate measurements of oxyhemoglobin and deoxyhemoglobin. The concentrations of $\mathrm{COHb}$ and $\mathrm{MetHb}$ in the artery were developed with pulse oximetry. ${ }^{8)}$ However, method for obtaining the concentrations of $\mathrm{Hb}$ derivatives for whole tissue was not developed. We have studied the measurement of the absolute values of $\mathrm{Hb}$ derivative concentrations using spatially resolved NIRS. Although extinction coefficients of $\mathrm{COHb}$ and MetHb were reported, ${ }^{9)}$ photon propagation in a turbid media contained $\mathrm{COHb}$ and MetHb was not adequately analyzed. We have examined by Monte Carlo simulation and experiments using eight wavelength NIRS system. Figure 7 shows the spatial slope of light intensities detected at $20 \mathrm{~mm}$ and $30 \mathrm{~mm}$ from the light source on experiments with phantoms contained $\mathrm{COHb}$ and $\mathrm{O}_{2} \mathrm{Hb}$. The 


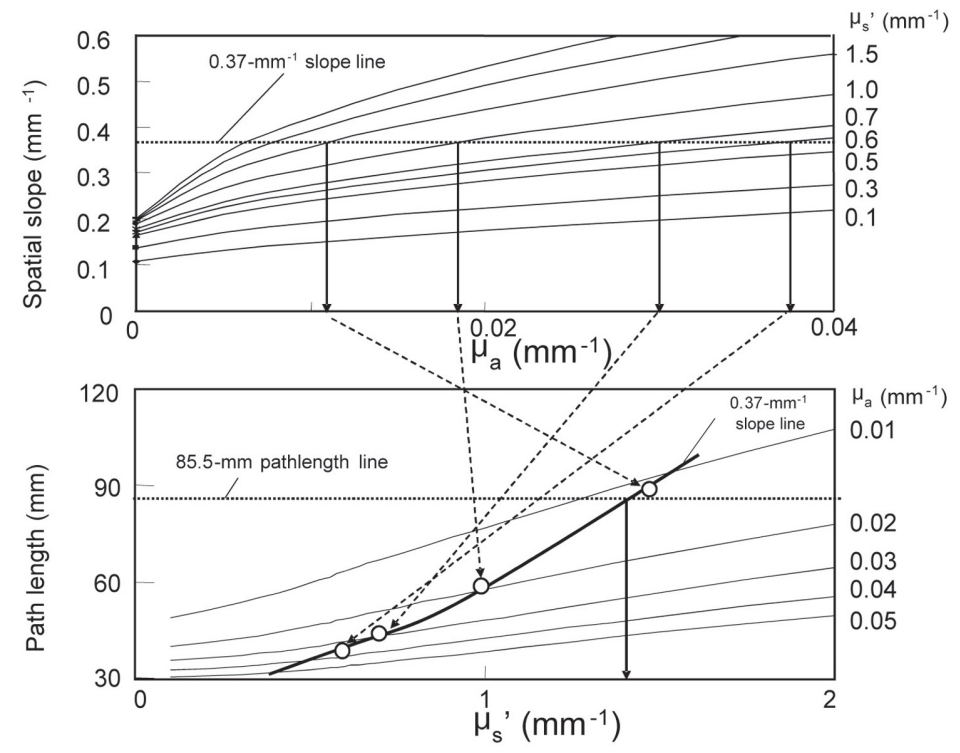

Figure 6 Determination of the optical properties using two graphs.

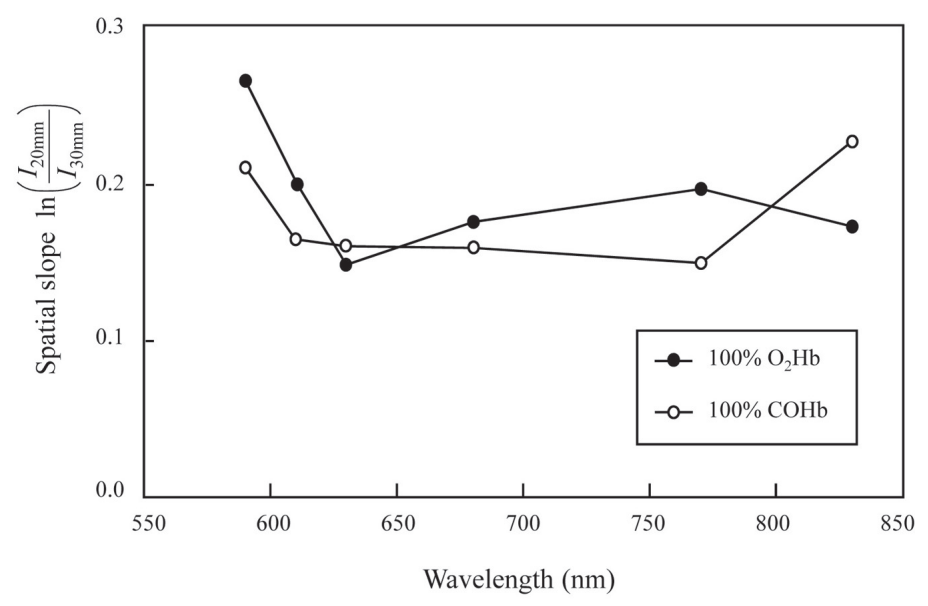

Figure 7 Relationship between wavelength and spatial slope for the phantoms contained $\mathrm{O}_{2} \mathrm{Hb}$ and $\mathrm{COHb}$.

absolute values of concentrations of $\mathrm{COHb}$ and $\mathrm{O}_{2} \mathrm{Hb}$ can be first determined by calculating the spatial slope at two wavelengths of $600-730 \mathrm{~nm}$. These results suggest that spatially resolved measurement at appropriate wavelengths would be useful to quantify the absolute value of $\mathrm{Hb}$ derivative concentrations.

\section{Conclusion}

We have shown the influences of fat thickness, skin oxygenation, scattering coefficient of surface layer, and $\mathrm{Hb}$ derivatives on accuracy in NIRS measurement. To improve the accuracy in oxygenation measurement, combination of corrections of these influences will be important. Although the size of instrumentation for scattering coefficient measurement was $30 \mathrm{~cm}$, all electronic circuits for corrections would be embedded into severalcentimeter optical probe in the future study. Simultaneous corrections of the influences enable us to measure accurate oxygenation with CW-NIRS and spatially resolved NIRS.

\section{References}

1) Nossal R, Kiefer J, Weiss GH, et al: Photon migration in lay- 
ered media. Appl Opt 1988; 27: 3382-3391

2) Kienle A, Patterson MS, Dögnitz N, et al: Noninvasive Determination of the Optical Properties of Two-Layered Turbid Media. Appl Opt 1998; 37: 779-791

3) Yamamoto K, Niwayama M, Lin L, et al: Accurate NIRS measurement of muscle oxygenation by correcting the influence of a subcutaneous fat layer. Proc SPIE 1998; 3194: 166173

4) Niwayama M, Lin L, Shao J, et al: Quantitative measurement of muscle hemoglobin oxygenation using near-infrared spectroscopy with correction for the influence of a subcutaneous fat layer. Rev Sci Instrum 2000; 71: 4571-4575

5) Niwayama M, Sone S, Murata H, et al: Errors in muscle oxygenation measurement using spatially-resolved NIRS and its correction. J Jpn Coll Angiol 2007; 47: 17-20
6) Suzuki H, Niwayama M, Yamakawa T, et al: Simultaneous determination of absorption coefficients for skin and muscle tissues using spatially resolved measurements. Adv Mater Res 2011; 222: 309-312

7) Yamashita $T$, Niwayama M, Yamakawa $T$ : Accurate and fast estimation of optical properties using optical path length and spatially resolved reflectance measurement. Adv Mater Res 2011; 222: 318-321

8) Barker SJ, Curry J, Redford D, et al: Measurement of carboxyhemoglobin and methemoglobin by pulse oximetry: a human volunteer study. Anesthesiology 2006; 105: 892-897

9) van Kampen EJ, Zijlstra WG: Spectrophotometry of hemoglobin and hemoglobin derivatives. Adv Clin Chem 1983; 23: 200-257 